\title{
Temporomandibular disorder in the oral and maxillofacial fields
}

\author{
Young-Kyun Kim, D.D.S., Ph.D. \\ Editor-in-Chief of JKAOMS \\ Department of Oral and Maxillofacial Surgery, Section of Dentistry, Seoul National University Bundang Hospital, Seongnam, Korea
}

Temporomandibular disorder (TMD) is defined as orofacial pain and craniomandibular disorder. In short, it is a complex disorder related to temporomandibular joint (TMJ), masticatory muscle, and occlusion ${ }^{1}$. TMD is known as a multifactorial disease and is difficult to diagnose and treat because it has various clinical symptoms and etiology and the causal factor has yet to be defined ${ }^{2}$. In the past, irreversible treatments such as surgical, orthodontic, and prosthodontic therapy were performed according to only one theory, but many complications arose, with no good results. There are many etiologies of TMD. Researchers who point to disc displacement as a main reason are interested in manipulation therapy, anterior repositioning splint, and surgical therapy such as meniscoplasty. On the other hand, researchers who focus on synovial pathology concentrate on joint injection, arthrocentesis, and arthroscopic surgery. Note, however, that TMD occurs for many reasons; hence the need to apply various treatment methods instead of using only one method.

The incidence of TMD increases when joint is consistently overloaded. Psychological factor, oral parafunction such as clenching, bruxism, and unilateral chewing, malocclusion, and maxillofacial trauma cause trauma on TMJ directly or indirectly; thus causing $\mathrm{TMD}^{3}$. Many studies were conducted to identify the reason for and etiology of $\mathrm{TMD}^{4-9}$. Note, however, that they have yet to be discovered, and TMD is one of the uncovered areas in dentistry. An unknown field can be very interesting for researchers with its many themes to be researched. Therefore, TMD is an item to be researched actively by oral and maxillofacial surgeons. In the past when surgical treatment was carried out actively, many surgeons were interested, giving presentations in conferences and publishing many articles. When the indication of surgical therapy became very narrow and conservative, and semisurgical treatments showed good results, however, surgeons lost interest. Nowadays, presentations on TMD in oral and maxillofacial surgery conferences or journals are very few and are on the decline.
A specific treatment method for TMD is not yet established, and it is impossible to conclude that there is a particularly good method; hence the need to select non-invasive, reversible treatment first. If pain and dysfunction continue, and conservative treatment does not work, prosthodontic, orthodontic, semi-surgical, or surgical treatment should be considered. The purposes of TMD treatment are decrease of pain and inflammation, decrease of muscle's hyperactivity, prevention of mechanical overload on muscle and joint, and restoration of jaw function. There is a need to reestablish oral and maxillofacial surgeons' concept of TMD based on these recent treatments. In other words, to treat TMD, we must understand that non-surgical conservative treatments such as counseling, medication, physical therapy, or splint therapy are required, and that we should pay attention to them. Oral and maxillofacial surgeons who consider only surgical therapy cannot hope to treat TMD.

\section{References}

1. Okeson JP. Current terminology and diagnostic classification schemes. Oral Surg Oral Med Oral Pathol Oral Radiol Endod 1997; 83:61-4.

2. Kim YK, Kim HT, Kim IS. Effect of initial conservative treatment of TMD patients: counseling and medication. J Korean Dent Assoc 2000;38:549-57.

3. Yun PY, Kim YK. The role of facial trauma as a possible etiologic factor in temporomandibular joint disorder. J Oral Maxillofac Surg 2005;63:1576-83.

4. Kim SG, Yeo HH, Kim YK, Kang DW. A clinical study on TMJ rthrocentesis. J Korean Assoc Oral Maxillofac Surg 1997;23:673-8.

5. Kim YK, Yeo HH, Cho SI, Kim MS. Enzymeimmuno-assay of PCE2 in the synovial fluid from TMJ of the patients with mandibular condylar fracture and internal derangement. J Korean Assoc Oral Maxillofac Surg 1996;22:65-9.

6. Choi EA, Lee DG, Chae CH, Chang YI, Park YJ, Kim YK. Preliminary study on the effect of inflamed TMJ synovial fluid on the intracellular calcium concentration and differential expression of iNOS and COX-2 human immortalized chondrocyte C28/I2. J Korean Assoc Oral Maxillofac Surg 2006;32:36-41.

7. Kim YK, Kim SG, Kim BS, Lee JY, Yun PY, Bae JH, et al. Analysis of the cytokine profiles of the synovial fluid in a normal temporomandibular joint: preliminary study. J Craniomaxillofac Surg 2012;40:e337-41. 
8. Kim YK, Kim SG, Im JH, Yun PY. Clinical survey of the patients with temporomandibular joint disorders, using Research Diagnostic Criteria (Axis II) for TMD: preliminary study. J Craniomaxillofac Surg 2012;40:366-72.
9. Kim BS, Kim YK, Yun PY, Lee E, Bae J. The effects of estrogen receptor $\alpha$ polymorphism on the prevalence of symptomatic temporomandibular disorders. J Oral Maxillofac Surg 2010;68: 2975-9. 